\title{
Cartographie de L'accessibilité et Gouvernance Locale des Infrastructures Hydrauliques dans la Commune D’avrankou Au Sud-Est du Benin
}

\author{
Zannou Sandé, \\ Laboratoire de l'Aménagement du Territoire, de l’Environnement et de \\ Développement Durable (LATEDD) \\ Dossou Guedegbe Odile, \\ Département de Géographie et Aménagement du Territoire/ FASHS/ \\ Université d’Abomey-Calavi (UAC)
}

Doi:10.19044/esj.2021.v17n37p269

Submitted: 31 August 2021

Accepted: 20 October 2021

Published: 31 October 2021
Copyright 2021 Author(s)

Under Creative Commons BY-NC-ND 4.0 OPEN ACCESS

Cite As:

Sandé Z. \& Odile D. G (2021). Cartographie De L'accessibilité Et Gouvernance Locale Des Infrastructures Hydrauliques Dans La Commune D'avrankou Au Sud-Est Du Benin. European Scientific Journal, ESJ, 17(37), 269-286.

https://doi.org/10.19044/esj.2021.v17n37p269

\section{Resume}

Dans la Commune d’Avrankou, les populations éprouvent d'énormes difficultés pour s'approvisionner en eau malgré la disponibilité d'une importante quantité de points d'eau. Ce travail vise à analyser la répartition des infrastructures hydrauliques et leur mode de gestion dans ladite Commune. Pour cartographier la disponibilité des points d'eau et étudier leur mode de gestion, 115 ménages et 17 personnes ressources ont été interrogés. Le logiciel Arc GIS 10.5 a été utilisé pour la spatialisation des données. Les résultats révèlent que la Commune d'Avrankou dispose de 301 infrastructures hydrauliques dont 83 fonctionnelles et 218 non fonctionnelles. Les points d'eau fonctionnels sont constitués de 42 Forages équipés de Pompe à Motricité Humaine et 41 Bornes Fontaines alimentées par 2 Adductions d'Eau Villageoise. Sur les 84 villages de la Commune, seulement 39 villages ont accès aux points d'eau fonctionnels soit 46,42 \% et parmi eux, 12 villages y ont doublement accès. Ainsi, 45 villages auraient accès si les autorités réparaient les points d'eau non fonctionnels et 15 villages si on construisait d'autres ouvrages. Les modes de gestion des infrastructures hydrauliques adoptées sont l’affermage et la gestion délégataire. Ces modes de gestion ont 
permis à la mairie de mobiliser des ressources pour financer les actions de développement. L'inégale répartition des infrastructures hydrauliques est la preuve de l'inexistence d'un système de maintenance régulier et de l'absence des outils de planification spatiale dans ce secteur. Pour y remédier, il faut donc impliquer davantage et responsabiliser les populations pour la gestion des points d'eau et réparer également les ouvrages hydrauliques non fonctionnels.

Palabras Clave: Avrankou, Cartographie, infrastructures hydrauliques, accessibilité, gouvernance locale

\title{
Mapping of Accessibility and Local Governance of Hydraulics Infrastructures in The Municipality of Avrankou, South-East of Benin
}

\author{
Zannou Sandé, \\ Laboratoire de l'Aménagement du Territoire, de l'Environnement et de \\ Développement Durable (LATEDD) \\ Dossou Guedegbe Odile, \\ Département de Géographie et Aménagement du Territoire/ FASHS/ \\ Université d'Abomey-Calavi (UAC)
}

\begin{abstract}
In the Municipality of Avrankou, the populations experience enormous difficulties in obtaining water supplies despite the availability of a large number of water points. This work aims to analyze the distribution of hydraulic infrastructures and their mode of management in the said Municipality. To map the availability of water points and study their management method, 115 households and 17 resource people were interviewed. Arc GIS 10.5 software was used for the spatialization of the data. The results reveal that the Municipality of Avrankou has 301 hydraulic infrastructures, of which 83 are functional and 218 are non-functional. The functional water points are made up of 42 Boreholes equipped with Human Motricity Pumps and 41 Fountains supplied by 2 Village Water Adductions. Out of the 84 villages of the Commune, only 39 villages have access to functional water points, either $46.42 \%$ and among them, 12 villages have double access to it. Thus, 45 villages would have access if the authorities repaired non-functional water points and 15 villages if other structures were built. The management methods of the hydraulic infrastructures adopted are leasing and delegated management. These management methods have enabled the town hall to mobilize resources to finance development actions. The
\end{abstract}


unequal distribution of hydraulic infrastructure is proof of the lack of a regular maintenance system and the absence of spatial planning tools in this sector. To remedy this, it is therefore necessary to involve the populations more and make them responsible for the management of water points and also to repair non-functional hydraulic structures.

Keywords: Avrankou, Cartography, Hydraulic Infrastructures, Accessibility, Local Governance

\section{Introduction}

Omniprésente et rare, l'eau est l'une des ressources les plus essentielles de la planète terre. Selon F. Azonsi (2009, p. 3), « elle est une ressource fondamentale des activités humaines, un bien commun de l'humanité auquel un accès équitable doit être garanti ». C'est un bien précieux plus indispensable que l'or, le pétrole ou autres ressources utiles à l'homme. Mais, « les changements climatiques et les processus migratoires soulèvent de nouveaux défis pour l'accès à l'eau car la concurrence pour les ressources en eau à des fins agricoles augmente » (C. Baron et A Bonnassieux, 2008, p. 18). Ainsi, d'après Solidarité Internationale (2020, p. 5) «plus de 2,2 milliards de personnes soit $29 \%$ de la population mondiale n’ont pas accès à l'eau ». En fait, « la consommation d'eau dans le monde a plus que triplé en cinquante ans, en raison de la croissance démographique importante et des besoins croissants de la population en eau » (L. Odounlami et al., 2012, p. 568).

Au Bénin, l'accès à l'eau est critique surtout en milieu rural. La gestion des ressources en eau, caractérisée par une multiplicité des centres de décision, une faible implication des acteurs et des usagers dans la prise de décision et la gestion des conflits entre agriculteurs et pasteurs sont à la base des difficultés de gestion des ressources en eau. Selon S. W. Boko (2009, p. 49), "le problème de gestion des ressources en eau par les autorités locales se pose avec acuité et occupe désormais une partie importante des débats de la Gestion Intégrée des Ressources en Eau (GIRE) ». Avec la décentralisation, c’est la collectivité locale qui prend en charge la gouvernance des ressources en eau. Selon F. Kpohonsito (2007, p.64), « la gouvernance locale des ressources en eau combine une action collective entre la population et les autorités locales ». L'accessibilité de la population à l'eau est donc le résultat d'une bonne gouvernance.

La Commune d'Avrankou ne fait pas exception aux difficultés d'accès à l'eau potable en dépit des infrastructures hydrauliques réalisées par la Mairie. D'un arrondissement à un autre, l'ampleur du problème semble varier. La présente recherche vise à analyser le niveau d'accessibilité et le mécanisme de gouvernance locale des infrastructures hydrauliques dans la Commune d'Avrankou. 


\section{Milieu D’Étude}

La Commune d'Avrankou est située au Sud-est du Bénin dans le département de l'Ouémé, entre $6^{\circ} 31^{\prime}$ et $6^{\circ} 38^{\prime}$ ' de latitude Nord et entre $2^{\circ} 36^{\prime}$ et $2^{\circ} 42^{\prime}$ de longitude Est. Elle est limitée au Nord par la Commune de Sakété, au sud par les Communes d'Adjarra et de Porto-Novo, à l'Est par la Commune d’Ifangni et la République Fédérale du Nigeria, à l'Ouest par la Commune d’Akpro-Missérété (figure 1).

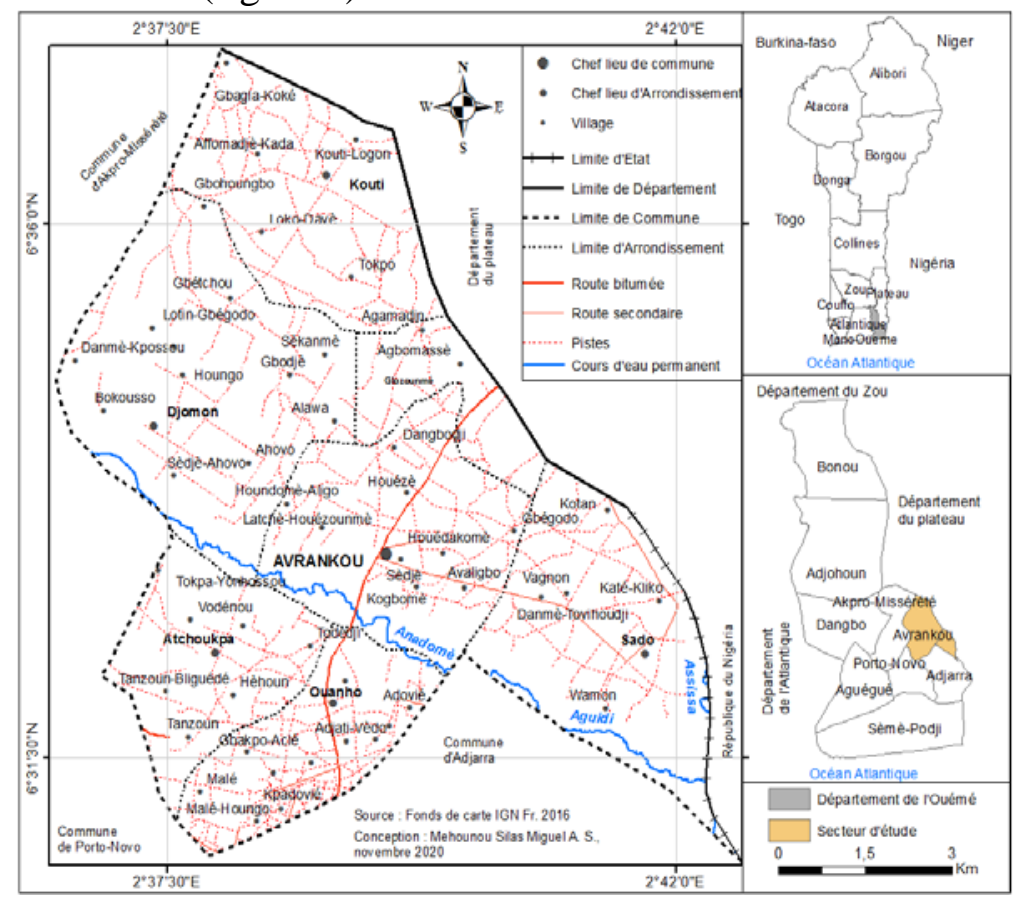

Figure 1: Situation géographique de la Commune d’Avrankou

La Commune d'Avrankou compte sept (07) arrondissements qui sont : Atchoukpa, Avrankou, Djomon, Gbozounmè, Kouti, Ouanho et Sado. Elle regroupe quatre vingt quatre villages et quartiers répartis par arrondissement avec une population totale de 94651 habitants en 2019 (INSAE, 2020, p 36). Cette forte population implique un besoin de plus en plus accru en ressource en eau. Le climat de la Commune de Avrankou est du type subéquatorial et bimodal à deux saisons pluvieuses et deux saisons sèches avec une pluviométrie annuelle qui se situe entre 1400 et $1500 \mathrm{~mm}$. Ce climat est favorable à la disponibilité des eaux de pluie et de surface.

\section{Matériels Et MÉthodes}

\subsection{Typologie des données collectées sur le terrain}

Les données collectées et utilisées pour atteindre l'objectif de cette recherche sont : (i) la liste des points d'eau et leur statut (fonctionnel ou non) obtenue au cours des enquêtes de terrain. Ceci a permis de connaître le nombre 
et les types des sources d'approvisionnement en eau de la Commune ; (ii) les coordonnées géographiques de chaque source prises avec l'application locus map pour l'analyse spatiale de la distribution des infrastructures hydrauliques ; (iii) les modalités d'accès de la population par type d'infrastructure pour identifier les localités ayant accès à l'eau ; (iv) les modes de fonctionnement de chaque catégorie de sources d'approvisionnement en eau pour connaître les difficultés inhérentes à leur fonctionnement; (v) les acteurs et les modes de gestion ; (vi) les difficultés et les contraintes liées à la bonne gestion des infrastructures hydrauliques dans la Commune.

\subsection{Echantillonnage et technique de collecte des données}

La Commune compte 7 arrondissements et 84 villages. Tous les arrondissements ont été systématiquement parcourus pour recenser les infrastructures hydrauliques et les enquêtes auprès des ménages ont été faites dans 14 villages. Pour déterminer la taille de l'échantillon, la méthode de Schwartz (1995) a été utilisée avec un degré de confiance de $95 \%$ et une marge d'erreur de plus ou moins $5 \%$. Elle se traduit par l'expression : $\mathrm{N}=$ $\mathrm{Z} \alpha^{2} . \mathrm{PQ} / \mathrm{d}^{2}$

avec : $\mathrm{N}=$ taille de l'échantillon par arrondissement ; $\mathrm{Z} \alpha=$ écart fixé à 1,96 correspondants à un degré de confiance de $95 \%$; $\mathrm{P}=$ rapport nombre de ménages des villages retenus/nombre de ménages total de la Commune d'Avrankou ; Q = 1-P ; d = marge d'erreur qui est égale à $5 \%$. Au total, 115 ménages ont été questionnés.

Les 115 personnes interrogées étaient exclusivement des femmes. Le choix exclusif des femmes se justifie par le fait que ce sont elles qui s'occupent de l'approvisionnement des ménages en eau. Les femmes choisies étaient spécifiquement celles qui sont mariées, âgées d'au moins vingt (20) ans et ayant au moins un enfant. En plus de ces enquêtées, huit (08) autorités locales (3 chefs d'arrondissement et 5 Chefs villages), le SG de la mairie, le chef service planification et développement local), un (01) responsable des services Eau et Assainissement et deux (02) fermiers en charge des infrastructures hydrauliques ont été interviewés.

Pour collecter les données et informations, diverses techniques ont été utilisées. L'observation directe a été utilisée pour faire l'inventaire systématique et la prise des coordonnées géographiques des infrastructures hydrauliques. L'enquête par questionnaire a permis de recueillir auprès des femmes, les données quantitatives sur les sources d'approvisionnement en eau dans les ménages. L'entretien avec les personnes ressources a permis de collecter les données qualitatives relatives aux modes de gestion des infrastructures hydrauliques, aux facteurs limitant l'accès des populations aux infrastructures hydrauliques fonctionnels, etc. 


\subsection{Traitement des données et analyse des résultats}

Le traitement des données a consisté au dépouillement et à la codification des données issues des fiches d'enquêtes. Les données ont été regroupées par catégories et par nature afin de calculer de façon automatique les fréquences des réponses. Les coordonnées géographiques des points d'eau prises au moyen de l'application locus map ont été transférées à l'ordinateur sur une feuille de calcul Excel 2013 via le logiciel Map source. Ces données ont permis de catégoriser les infrastructures hydrauliques dans la Commune. Ensuite, ces coordonnées ont été ouvertes et superposées aux données vecteurs (shapfile) de base, comme : la limite administrative de la Commune, les routes et les localités en vue de produire la carte de répartition des infrastructures hydrauliques. Enfin, à partir de ces infrastructures, du positionnement des villages et des distances qui les séparent des points d'eau, le niveau d'accessibilité de la population à l'eau a été analysé par la production de la carte d'accessibilité.

L'analyse spatiale est basée sur les normes requises pour la répartition des infrastructures hydrauliques et sur les résultats des investigations en milieu réel. Selon l'OMS (1992, p.47), ces normes sont de un point d'eau pour 250 habitants avec une couverture de $200 \mathrm{~m}$ de rayon. Ceci a permis d'identifier les villages qui ont accès à l'eau et les villages qui n’y ont pas accès. Ces différentes opérations ont été faites avec le logiciel Arc gis 10.5. Cette même analyse a été faite pour les ouvrages hydrauliques non fonctionnels dans le but de faire des propositions de réparation de ces ouvrages afin de réduire un tant soit peu l'inaccessibilité à certaines sources d'eau dans cette Commune.

\section{RÉSULTATS}

\subsection{Typologie et répartition des infrastructures hydrauliques dans la Commune d'Avrankou}

Dans la Commune d'Avrankou, on distingue deux types d'infrastructures hydrauliques réalisées par les pouvoirs publics. Il s'agit des Forages équipés de Pompe à Motricité Humaine (FPM) et des Adductions d'Eau Villageoise (AEV). Dans la Commune, deux types de FPM sont enregistrés. Il s'agit de la pompe AFRIDEV, une pompe à bras (10 à $30 \mathrm{~m}$ de profondeur) et de la pompe VERGNET qui est une pompe à pied (40 à $70 \mathrm{~m}$ de profondeur). Au total, 177 FPM couvrent la Commune d'Avrankou. La photo 1 montre un forage fonctionnel dans le village de Dangbodji. 


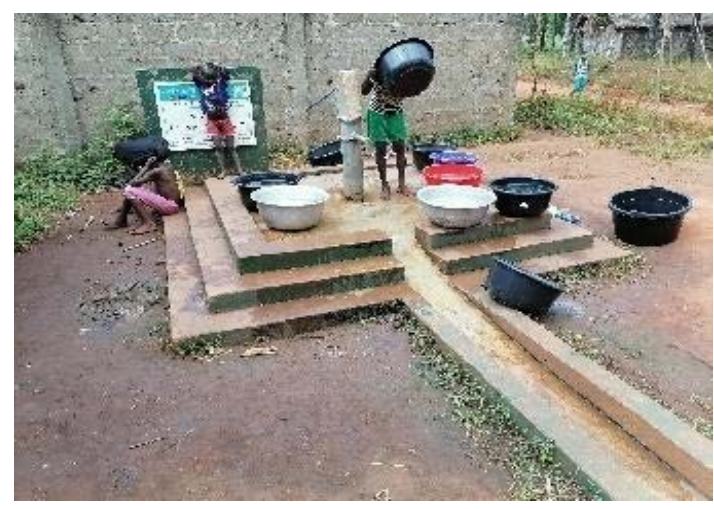

Prise de vue. Zannou, décembre 2020

Photo 1. Forage équipé de Pompe à Motricité humaine à Dangbodj

L'observation de la photo 1 montre que le forage est très exploité par la population. La Commune dispose de 177 forages dont 58 en panne et 77 abandonnés et hors parc. Ainsi, seulement 42 forages sont fonctionnels dans la Commune, soit $23,73 \%$. Seulement $41,74 \%$ des ménages enquêtés s'approvisionnent au niveau des forages. Les forages sont inégalement répartis dans la Commune. La figure 2 présente la répartition spatiale des FPM dans la Commune.

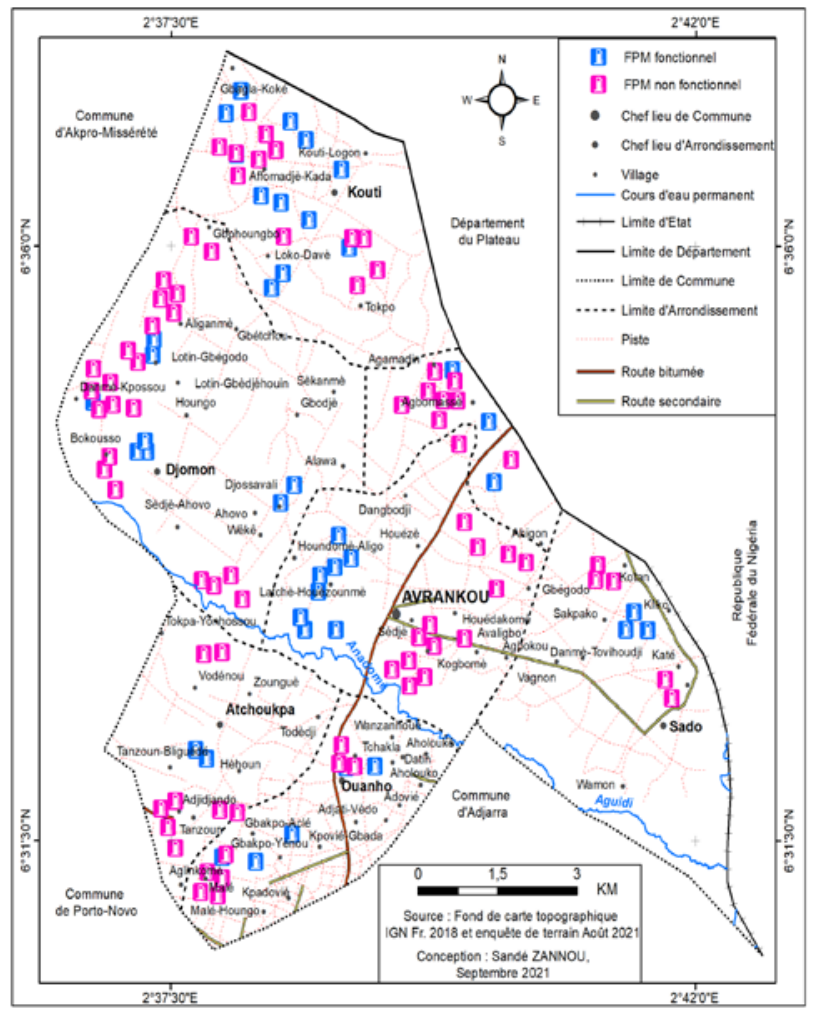

Figure 2. Répartition spatiale des FPM dans la Commune d'Avrankou 
L'analyse de la figure 2 montre que les forages sont inégalement répartis dans la Commune. En effet, l'arrondissement de Atchoukpa dispose de 16 FPM dont seulement 2 fonctionnels. Les arrondissements d'Avrankou et Djomon disposent respectivement de 8 FPM fonctionnels. Dans l'arrondissement de Gbozounmè, 4 forages sont fonctionnels. A Kouti, 12 FPM fonctionnent sur les 34 disponibles. Dans l'arrondissement de Ouanho, 5 FPM fonctionnels sur les 22 disponibles. Seulement 3 FPM fonctionnent dans l'arrondissement de Sado.

Les enquêtes de terrain ont révélé que les forages ont été financé par des organisations et partenaires internationaux de développement. Il s'agit par exemple de la Banque Mondiale et de la GIZ qui restent les principaux Partenaires Techniques et Financiers de la Commune. Leur contribution a permis la réalisation de 7 Adductions d'Eau Villageoise (AEV) dans la Commune. Une AEV est composée d'un château d'eau qui représente le réservoir d'eau et d'un groupe électrogène (photo 2).

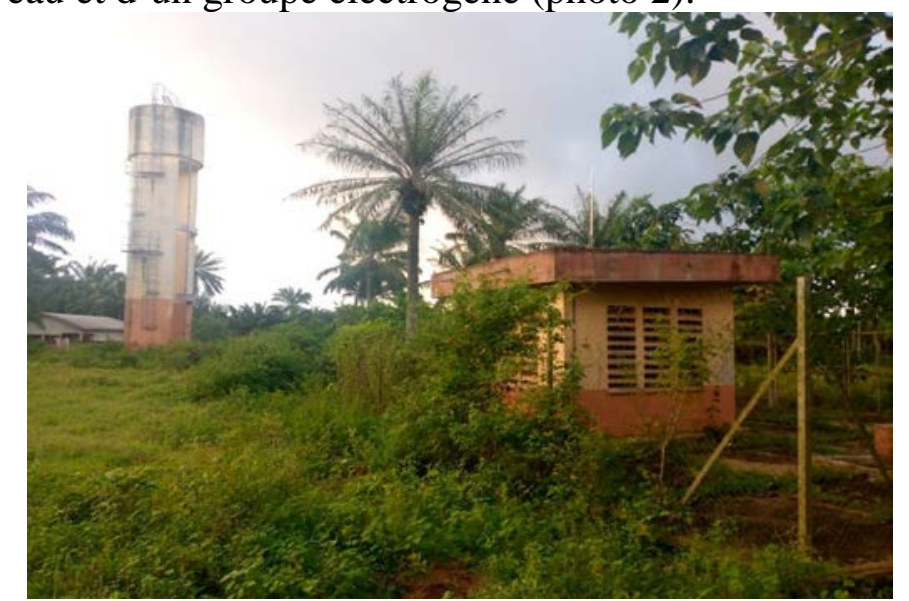

Prise de vue. Zannou, décembre 2020

Photo 2. Dispositif de l'Adduction d'Eau Villageoise (AEV) de Seligon

La photo 2 présente le système d'AEV composé du château d'eau et du moteur de pompage réalisé à Seligon dans l'arrondissement de Gbozounmè. La distribution de l'eau stockée dans le château se fait par les Bornes Fontaines (BF). Les AEV et les BF sont inégalement répartis dans la Commune. L'absence d'un outil de planification des ouvrages hydrauliques explique les disparités en infrastructures hydrauliques qui existent. La figure 3 présente la répartition spatiale des $\mathrm{AEV}$ et des $\mathrm{BF}$ dans la Commune. 


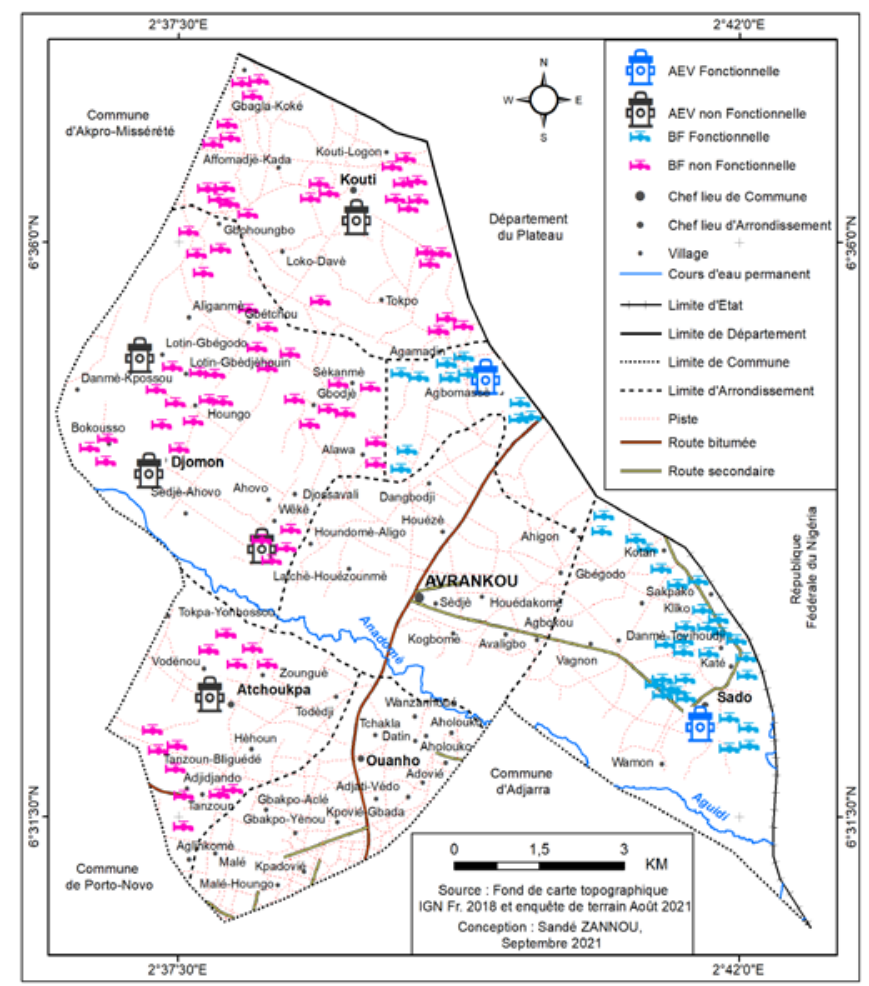

Figure 3. Répartition spatiale des AEV et des BF dans la commune d'Avrankou

Il ressort de l'analyse de la figure 3 que 2 AEV sont fonctionnelles sur les $7 \mathrm{AEV}$ que dispose la Commune. Seuls les arrondissements de Gbozounmè et de Sado disposent chacun d'une AEV fonctionnelle. Sur les 124 Bornes Fontaines (BF), seulement $41 \mathrm{BF}$ sont fonctionnelles, à raison de $11 \mathrm{BF}$ dans l'arrondissement de Gbozounmè et $30 \mathrm{BF}$ dans l'arrondissement de Sado. Ces BF sont des points d'eau publics qui permettent la distribution des eaux des AEV. L'inégale répartition et le statut non fonctionnel de certaines infrastructures hydrauliques dans la commune créent des problèmes d'accessibilité. Seulement 22,60 \% des ménages enquêtés utilisent l'eau des Bornes Fontaines dans les activités domestiques.

\subsection{Gestion des infrastructures hydrauliques dans la Commune}

La gestion des ouvrages hydrauliques (Adductions d'Eau Villageoises et forages) permet la mobilisation des ressources pour le financement du développement local dans la commune. Selon les enquêtes de terrain, le mode de gestion des Adductions d'Eau Villageoises (AEV) dans la Commune jusqu'en 2008 est la régie autonome, qui est dotée de l'autonomie financière et dispose d'un organe de gestion individualisée. Les AEV sont gérées par trois catégories d'acteurs : les Associations de Développement Villageois, le comité de gestion et l'exploitant. A ces acteurs, sont associés les gestionnaires 
des bornes fontaines. Les forages étaient gérés par un comité mis en place par les populations en collaboration avec la direction générale de l'hydraulique.

A partir de 2009, les autorités communales ont adopté la gestion déléguée des ouvrages hydrauliques. Ainsi, les AEV sont exploitées par le fermier (l'entreprise déléguée à la gestion) qui doit verser une redevance mensuelle à la Commune. Cette redevance est de $30 \mathrm{~F} / \mathrm{m}^{3}$ d'eau produite. L'AEV de Gbozounmè est gérée par le fermier CORPORET et celle de Sado est gérée par le fermier LIBRACEL. Dans la Commune, il a été dénombré 150 branchements privés au réseau de distribution d'eau des AEV. Ces ménages abonnés payent $300 \mathrm{~F} / \mathrm{m}^{3}$ d'eau. Les forages sont gérés par un gestionnaire qui est choisi au sein de la population par la communauté sous la supervision des autorités communales. Il assure la vente de l'eau et l'entretien de l'ouvrage. Les gestionnaires des forages versent une somme de $4000 \mathrm{~F}$ par mois dans le compte de la mairie. En cas de panne, seuls les artisans réparateurs de la commune ont le droit d'intervenir sur les forages. L'évolution des recettes issues de la gestion des infrastructures hydrauliques dans la Commune est présentée par la figure 4.

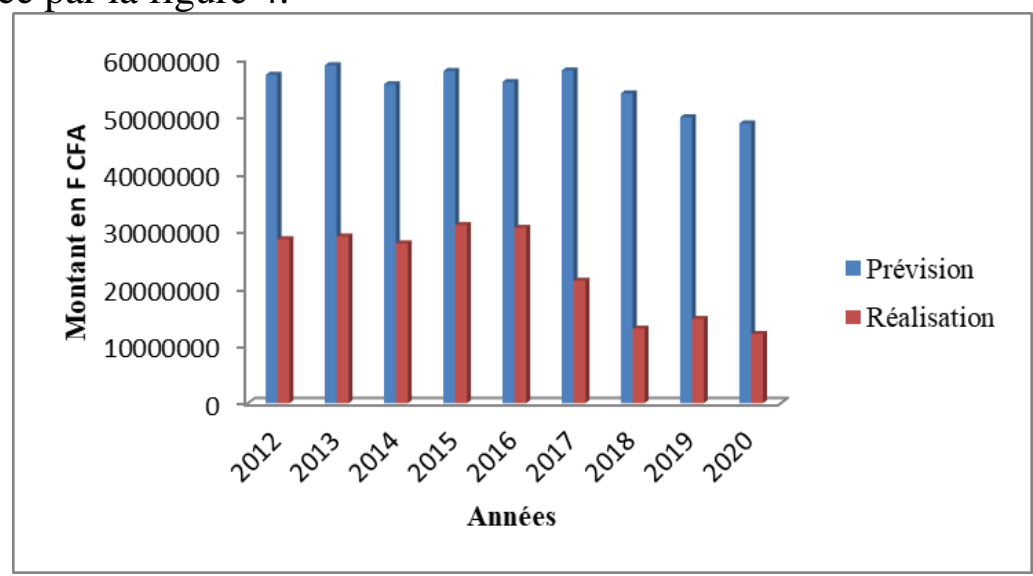

Source des données. Comptes administratifs de la Commune

Figure 4. Evolution des recettes annuelles du secteur de l'eau de 2012 à 2020

L'analyse de la figure 4 montre que les recettes collectées dans le secteur de l'eau ont connu une diminution de 2012 à 2020. La baisse remarquable des recettes s'explique par la mauvaise gestion des infrastructures. En effet, en cas de panne, les infrastructures hydrauliques sont abandonnées. Selon les personnes interrogées, l'abandon des infrastructures hydrauliques en pannes s'explique par le manque de suivi de la mairie $(68,70$ \% des enquêtés) et le non respect du contrat par les délégataires des forages et bornes fontaines (31,30 \% des enquêtés).

Pour les gestionnaires, la non réparation des ouvrages hydrauliques en pannes à temps est souvent dû au manque de moyens financiers, à l'absence de main d'œuvre qualifiée sur place et à l'indisponibilité à proximité des 
pièces de rechange. Pour eux, le prix de vente de l'eau ne leur permet pas de faire face à toutes les dépenses liées à la gestion des ouvrages hydrauliques et de prendre encore en charge les pannes qui nécessitent souvent de gros moyens financiers. En effet, la gestion déléguée implique chez le contractant des dépenses régulières notamment les salaires des employés, la redevance de la Mairie et l'entretien des points d'eau. Dès lors, il est récurrent de constater la démission des délégataires et le non-respect des contrats d'affermage. A ces problèmes s'ajoute le manque de suivi de la mairie. Dans la Commune, seulement $2 \mathrm{AEV}$ sont fonctionnelles sur les 7 réalisées et 42 forages fonctionnels sur les 177 forages réalisés. Cet état de chose crée de profondes disparités en infrastructures hydrauliques dans la Commune.

\subsection{Analyse de l'accessibilité aux infrastructures hydrauliques dans la Commune}

\section{$\checkmark$ Accessibilité selon la distance établie par l'OMS}

L'accès de la population à l'eau ou à une source d'approvisionnement en eau fonctionnelle dépend de deux critères essentiels. La distance et la quantité. La distance qui sépare un ménage ou un village d'un point d'eau varie en fonction de l'éloignement du village et de l'emplacement de la source. Cette accessibilité de la population aux points d'eau selon la distance proposée par l’OMS (200 m) est présentée par la figure 5.

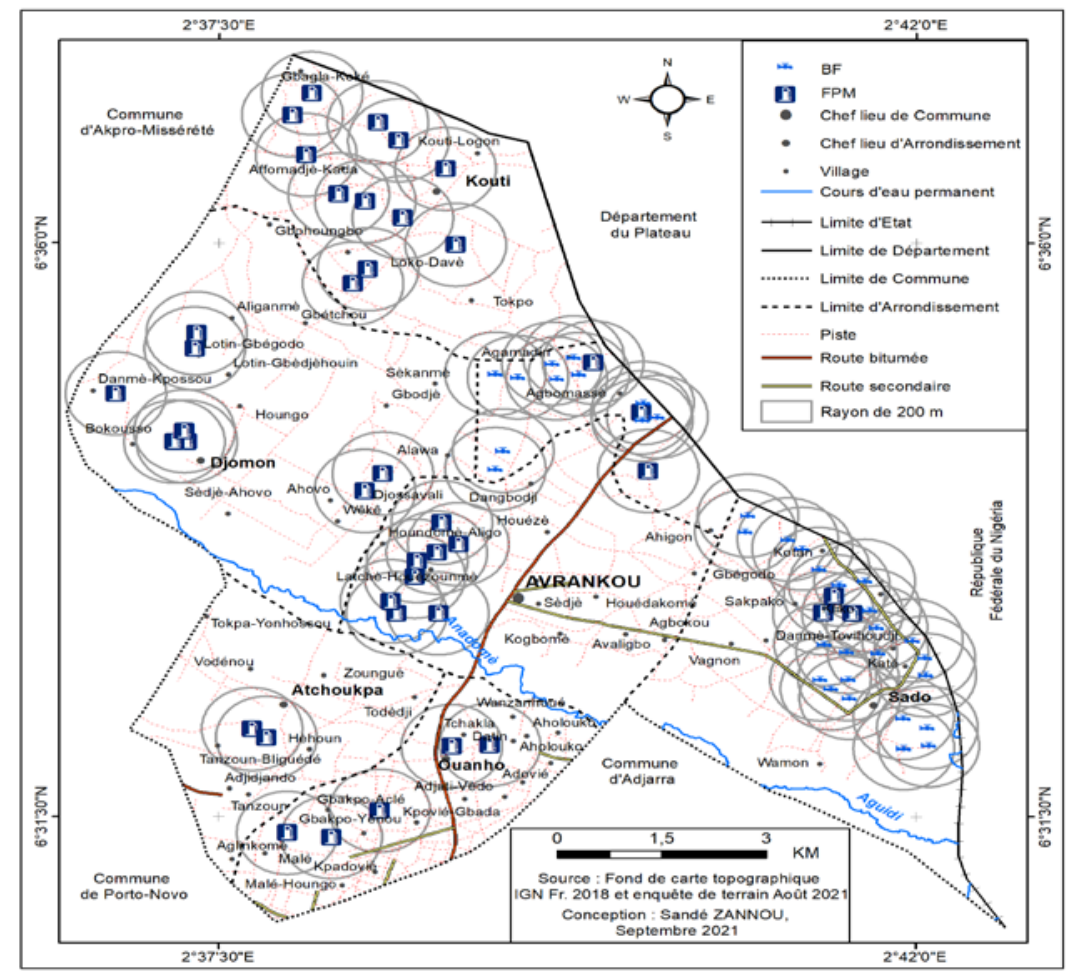

Figure 5. Accessibilité des points d'eau dans la Commune 
Il ressort de l'analyse de la figure 5 que sur les 84 villages de la commune, seulement 39 villages ont accès aux points d'eau fonctionnels soit $46,42 \%$. Il faut dire que certains villages ont accès, d'autres ont doublement accès pendant que d'autres n'ont pas du tout accès. En effet, dans l'arrondissement de Kouti, tous les villages ont accès aux points d'eau sauf le village de Tokpo. Seuls les villages de Kouti-Lognon et Gbegla-Koké ont accès à un point d'eau chacun, alors que les villages de Loko-Davè, Affomadjè-Kada et Kouti ont doublement accès aux points de FPM. Les villages Lotin-Gbegodo, Bokousso, Djomon Gbétchou (double accès), Aligamè, Lotin-Gbèdjèhouin et Danmè-Kpossou ont tous accès à au moins une source d'approvisionnement en eau dans l'arrondissement de Djomon. A Gbozoumè, tous les villages (Ahignon, Agamadin et Agbomassè) ont doublement accès aux BF et FPM dans l'arrondissement. Dans l'arrondissement d'Avrankou, les villages Latché-Houézoumè, HoundomèAligo et Dangbodji ont doublement accès aux sources d'eau que sont les FPM et les BF. Sur les 9 villages de l'arrondissement de Sado, seulement 03 villages n'ont pas accès aux sources d'eau. Les villages de Danmè-Tovihoudji, Sado, Sakpako, Kiliko, Katé et Kotan ont doublement accès aux sources d'eau. Les villages ayant accès aux points d'eau dans l'arrondissement d'Atchoukpa ont tous doublement accès aux points de FPM, sauf le village de Héhoun qui a accès à un seul point d'eau. Enfin, à Ouanho ce sont les villages de Ouanho et Tchakla qui ont doublement accès aux sources d'eau dans l'arrondissement. Il se pose un grand problème d'accessibilité à l'eau pour certaines localités sur la distance parcourue selon l'OMS.

La distance d'accessibilité (200 m) proposée par l'OMS appliquée aux infrastructures hydrauliques non fonctionnelles a permis d'identifier les villages qui auront accès si ces infrastructures fonctionnaient. La figure 6 présente l'accessibilité des infrastructures non fonctionnelles dans la Commune. 


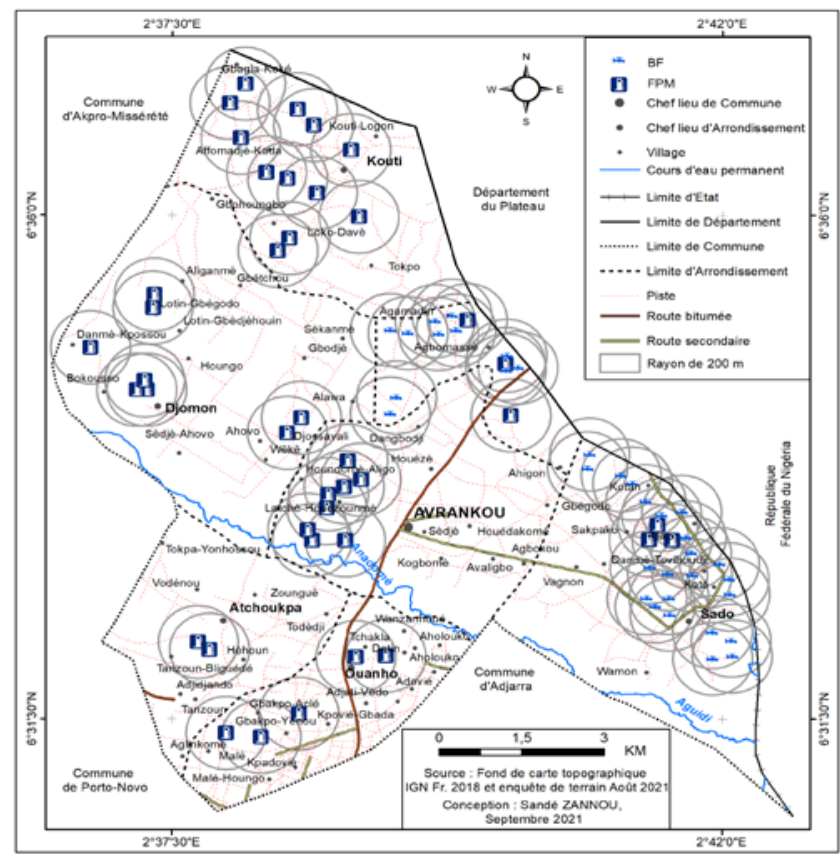

Figure 6. Accessibilité des villages aux infrastructures hydrauliques non fonctionnelles

Au total, sur les 84 villages de la commune, 45 villages pourraient avoir accès aux points d'eau non fonctionnels s'ils fonctionnaient soit 53,57 \% des villages. Par contre, même si ces points sont réparés et fonctionnaient, 15 villages n'auront pas toujours accès aux points d'eau. Ces villages sont Gbétchou, Sékanmè, Gbodjè, Houngo, Sèdjè-Ahovo, Houézè, TokpaYohonssou, Zounguè, Agbokou, Vagnon, Danmè-Tovihoudji, Wamon, Aholouko, Adovié, Adjati-Vèdo, Kpadovié et Malé-Houngo. Ce qui suppose non seulement qu'il faut réparer les points d'eau non fonctionnels, abandonnés mais également construire des ouvrages hydrauliques dans ces villages pour une couverture totale de la Commune.

\section{Accessibilité selon l'effectif de la population de chaque arrondissement}

Les tableaux 1 et 2 présentent la répartition du nombre des points d'eau fonctionnels et non fonctionnels en fonction de l'effectif de la population. Ceci a permis de faire l'analyse de l'accessibilité selon l'effectif de la population et par arrondissement en considérant que 250 habitants ont droit à 1 point d'eau. 
Tableau 1. Accessibilité de la population par arrondissement

\begin{tabular}{|c|c|c|c|c|c|c|}
\hline $\begin{array}{c}\text { Arrondis- } \\
\text { sement }\end{array}$ & $\begin{array}{c}\text { Population } \\
\mathbf{2 0 1 9}\end{array}$ & $\begin{array}{c}\text { Nombre total } \\
\text { de point } \\
\text { d'eau }\end{array}$ & $\begin{array}{c}\text { Point d'eau } \\
\text { fonctionnel }\end{array}$ & $\begin{array}{c}\text { Manquant } \\
\text { total }\end{array}$ & $\begin{array}{c}\text { Manquant } \\
\text { fonctionnel }\end{array}$ & $\begin{array}{c}\text { Manquant } \\
\text { non } \\
\text { fonctionnel }\end{array}$ \\
\hline Atchoukpa & 23323 & 29 & 2 & 93 & 91 & 66 \\
\hline Avrankou & 15179 & 31 & 8 & 61 & 53 & 30 \\
\hline Djomon & 16981 & 80 & 8 & 68 & 60 & +4 \\
\hline Gbozounmè & 7220 & 32 & 15 & 29 & 14 & 12 \\
\hline Kouti & 15200 & 62 & 12 & 60 & 48 & +2 \\
\hline Ouanho & 10385 & 22 & 5 & 42 & 37 & 20 \\
\hline Sado & 6363 & 45 & 33 & 25 & +8 & 13 \\
\hline Total & $\mathbf{9 4 6 5 1}$ & $\mathbf{3 0 1}$ & $\mathbf{8 3}$ & $\mathbf{3 7 8}$ & - & - \\
\hline
\end{tabular}

Source: Travaux de terrain, novembre 2020

Il ressort de l'examen du tableau 1 que les infrastructures hydrauliques sont inégalement reparties dans la Commune. L'arrondissement de Atchoukpa compte 23323 habitants, pour les couvrir en point d'eau, il faut réaliser 91 points d'eau si on considérait les 2 fonctionnels et il faut 66 points d'eau si on réparait les 27 non fonctionnels. Pour couvrir la population de l'arrondissement de Avrankou qui est estimée à 15179 habitants, il faut compléter 53 points d'eau aux 8 fonctionnels et disponibles et 30 points d'eau si les 23 non fonctionnels sont réparés. Quant à l'arrondissement de Djomon qui compte 80 points d'eau dont 08 sont fonctionnels, les 16981 habitants auront besoin de 60 points d'eau complémentaires en tenant compte des points fonctionnels et ils auront un surplus de 4 points d'eau si les 78 non fonctionnels sont réparés. A Gbozounmè, 32 points d'eau sont disponibles dont 15 sont fonctionnels. Les 7220 habitants auront besoins au total de 29 points d'eau de plus (14 points d'eau complémentaires en considérant les fonctionnels et 12 points d'eau si les non fonctionnels sont réparés). A Kouti, 62 points d'eau sont disponibles et 12 fonctionnent. Pour couvrir la population (15 200 habitants), il faut au total 60 points d'eau complémentaires (48 points d'eau complémentaires pour les fonctionnels et 2 de surplus si on réparait les non fonctionnels). L'arrondissement de Ouanho dispose de 22 points d'eau dont 5 seulement fonctionnent. Pour couvrir la population de cet arrondissement qui est estimée à 10385 habitants, il faut un complément de 42 points d'eau aux 5 points d'eau fonctionnels et 37 points d'eau si on réparait les non fonctionnels. Sado compte 6363 habitants avec 45 points d'eau dont 33 fonctionnent. A ce niveau, il y a un surplus de 8 points d'eau si on considérait les points d'eau fonctionnels et 13 si on réparait les non fonctionnels. Le tableau 2 présente le nombre de personnes ayant accès aux points d'eau par arrondissement 
Tableau 2. Accessibilité de la population par arrondissement

\begin{tabular}{|c|c|c|c|c|}
\hline Arrondissements & $\begin{array}{c}\text { Population } \\
\mathbf{2 0 1 9}\end{array}$ & $\begin{array}{c}\text { Point d'eau } \\
\text { fonctionnel }\end{array}$ & $\begin{array}{c}\text { Nombre } \\
\text { d'habitants } \\
\text { ayant accès }\end{array}$ & $\begin{array}{c}\text { Nombre } \\
\text { d'habitants } \\
\text { n'ayant pas accès }\end{array}$ \\
\hline Atchoukpa & 23323 & 2 & 500 & 22823 \\
\hline Avrankou & 15179 & 8 & 2000 & 13179 \\
\hline Djomon & 16981 & 8 & 2000 & 14981 \\
\hline Gbozounmè & 7220 & 15 & 3750 & 3470 \\
\hline Kouti & 15200 & 12 & 3000 & 12200 \\
\hline Ouanho & 10385 & 5 & 1250 & 9135 \\
\hline Sado & 6363 & 33 & 8250 & - \\
\hline Total & 94651 & 83 & 20750 & - \\
\hline
\end{tabular}

Source: Travaux de terrain, novembre à décembre 2020

L'examen du tableau 2 montre que le problème lié à l'accès aux points d'eau de consommation se pose avec acuité dans la Commune de Avrankou. Sur les 94651 habitants que compte la Commune en 2019, seulement 20750 habitants ont accès à une infrastructure hydraulique. Dans l'arrondissement de Sado, 8250 habitants ont accès aux points d'eau. Le problème de couverture en termes du nombre d'habitants ne se pose pas dans cet arrondissement. La situation est préoccupante dans les autres arrondissements de la Commune.

Pour palier aux problèmes d'eau dans la Commune, les populations réalisent elles-mêmes des postes d'eau autonomes (PEA). Il s'agit des ouvrages d'alimentation en eau comprenant un puits ou un forage et un système de pompage motorisé dont la pompe est entrainée par une source d'énergie électrique, un petit château et une pompe de robinets de distribution situés à proximité immédiate du forage ou du puits. A ces PEA, s'ajoutent aussi des puits traditionnels et semi-modernes qui constituent les infrastructures hydrauliques privées et personnelles rencontrées dans la Commune d'Avrankou.

\section{DiscusSiON}

Les résultats de cette recherche montrent que les points d'eau fonctionnels ne couvrent pas toute la Commune. Ils sont inégalement répartis dans tous les arrondissements de la Commune. Sur l'ensemble de ces points d'eau fonctionnels, seulement 28 villages sur 84 en ont accès et parmi eux, $42,85 \%$ ont doublement accès alors que 41 villages n'ont pas du tout accès. Cette inégale répartition a été signalée par GVP/AO (2009, p.12) au Niger dans le projet d'évaluation de la gouvernance de l'eau. Ces résultats s’apparentent également à ceux obtenus par B. Egounlety (2008, p. 22) qui en appliquant l'approche GIRE à la gestion des ressources en eau à l'échelle de la Commune de Zè, a diagnostiqué entre autres problèmes le caractère inéquitable des installations d'ouvrages en eau d'un arrondissement à un autre. Cette répartition inéquitable des infrastructures hydrauliques a été constatée 
par R. Bah-Agba (2014, p.39) dans son étude sur la gouvernance locale et l'approvisionnement en eau potable dans la Commune de Tchaourou.

En plus de l'inégale répartition des ouvrages, la gouvernance locale du secteur laisse entrevoir une disparité dans l'accessibilité des populations. En effet, des villages comme Loko-Davè, Gbétchou, Agbomassè, Vagnon, Vodénou et Hèhoun ont doublement accès aux points d'eau dans la Commune alors que d'autres villages n'ont accès à aucun ouvrage. Ainsi, il est noté la présence de plusieurs infrastructures hydraulique au même endroit. Ceci traduit la faiblesse de la gouvernance de l'eau dans cette Commune. Ces résultats confirment ceux présentés par D. Aholou-Houngnadji (2018, p.47) qui a montré que certains villages de la Commune de Adjohoun ont accès à plusieurs points d'eau potable à la fois pendant que d'autres en manquent. De même, ces résultats sont similaires à ceux obtenus par A. C. Adomou (2008, p.62) qui a mené une étude sur la décentralisation et la gouvernance de l'eau dans la Commune de Toffo. Il a montré que l'implantation des infrastructures hydrauliques ne tient pas compte du poids démographique et des pesanteurs sociologiques et culturelles. De plus chaque infrastructure, à Avrankou, devra supporter en moyenne 1140 habitants alors que, pour sa durabilité il lui faut supporter 250 habitants. Cette surexploitation explique les pannes répétées des infrastructures hydrauliques selon F. Kpohonsito (2007, p.53).

Les modes de gestion de ces ouvrages dans le secteur de recherche sont entre autres l'affermage et la gestion délégataire. Ces modes de gestion ont été mis en place dans toutes les Communes du Bénin d'après le MDAEP (2013, p.48) et ils impliquent plusieurs acteurs comme la mairie, les chefs d'arrondissement et la population. Le rôle déterminant de ces acteurs dans la gestion de l'eau a été également souligné par B. Hounmènou (2006, p.18). Mais contrairement à ces travaux, cette recherche met en exergue la défaillance des modes de gestion des ouvrages hydrauliques ainsi que l'absence des outils de planification spatiale de ces ouvrages comme étant les principales failles de la gouvernance locale qui affectent l'accessibilité à l'eau. Des résultats similaires ont été obtenus par R. Bah-Agba (2014, p.65) qui a montré que le non respect des contrats par les fermiers et l'absence d'un mécanisme de contrôle et de suivi dans la Commune de Tchaourou expliquent l'abandon des ouvrages hydrauliques en pannes. Par ailleurs, S. C. Hounguevou et al., (2014, p.226) ont montré que l'absence d'outil spatial dans la distribution des infrastructures hydrauliques est un grand facteur limitant l'accès à l'eau de consommation dans la Commune de Zè.

\section{Conclusion}

La présente recherche a présenté la problématique liée à la gouvernance et à l'accessibilité des infrastructures hydrauliques dans la Commune d'Avrankou. Elle a montré que la Commune dispose de 301 
infrastructures hydrauliques dont 83 fonctionnelles et 218 non fonctionnelles. De tout ce qui précède, l'on peut dire qu'il y a une défaillance dans la gouvernance locale des ouvrages hydrauliques, gérées par affermage pour certains et délégation communautaire pour d'autres. Cette défaillance notée dans les modes de gestion explique l'abandon de plusieurs ouvrages hydrauliques en pannes. Aussi, la disparité dans la répartition des ouvrages hydrauliques est-elle imputable à l'inexistence de véritables outils de planification et de gestion. Dès lors, il paraît plus que nécessaire que les autorités communales repensent la gouvernance du secteur de l'eau afin de combler véritablement les attentes liées à cette compétence à elles transférée à l'occasion de la décentralisation.

\section{References:}

1. Adomou, A. C. (2008). Décentralisation Et Gouvernance De L'eau En Milieu Rural Au Bénin : Cas De La Commune De Toffo, Département De L'atlantique. Diplôme D'etude Supérieure Spécialisée, Iub, Bénin, $80 \mathrm{P}$.

2. Aholou-Houngniadji, D. (2018). Gouvernance Locale Et Gestion Des Infrastructures Hydrauliques Dans La Commune D'adjahoun. Mémoire De Maîtrise De Géographie, Dgat/Flash/Uac, Bénin, 88 P.

3. Azonsi, F. (2009). Processus De Gestion Intégrée Des Ressources En Eau Au Bénin. Rapport D’atelier Sur La Gire, 21 P.

4. Bah-Agba, R. (2014). Gouvernance Locale Et Approvisionnement En Eau Potable Dans Les Milieux Ruraux Au Bénin: Cas De La Commune De Tchaourou. Mémoire De Maîtrise En Géographie, Université Du Québec À Rimouski, 193 P.

5. Baron, C. Et Bonnassieux, A. (2008). Accessibilité Aux Ressources En Eau Et Participation Des Acteurs Locaux : Quelles Réponses Faces Aux Enjeux De Durabilité Cas Des Associations D'usagers De L'eau $\mathrm{Au}$ Sud-Ouest Du Burkina-Faso. Journées Du Développement Du Gres, Burkina-Faso, $23 \mathrm{P}$

6. Boko, S. W. (2009). Gestion Communautaire Des Ressources En Eau Et Conflits D'usage Dans La Basse Vallée De L 'Ouémé. Diplôme D'etude Supérieure Spécialisée, Uac, Bénin, 62 P.

7. Egounlety, B. (2008). L'approche Gire Appliquée À La Gestion Des Ouvrages D'eau Potable À L'échelle Communale Dans Les Communes De Zè. Rapport De Formation, Niaouli, Bénin, 37 P.

8. Gvp/Ao, (2009). Evaluation De La Gouvernance De L'eau Au Niger. Rapport, 57 P.

9. Hounguevou, S. C. ; Tohozin, C. A.; Soumah, M. Et Toko Mouhamadou, I. (2014). Sig Et Distribution Spatiale Des 
Infrastructures Hydrauliques Dans La Commune De Zè Au Bénin. In Afrique Science, Issn 1813-548x, Pp. 213-227.

10. Hounmenou, B. (2006). Gouvernance De L'eau Potable Et Dynamique Locale En Zone Rurale $\mathrm{Au}$ Bénin. Http://Journals.Penedition. Org/Développementdurable/1763 ; Doi, 29 P.

11. Insae, (2020). Synthèses Des Résultats Et Estimation De La Population. Cotonou, Bénin, $41 \mathrm{P}$.

12. Kpohonsito, F. (2007). La Gestion Communale Des Ouvrages D'approvisionnement En Eau Potable En Milieu Rural Au Bénin : Cas De La Commune De Bopa. Mémoire De Maîtrise En Géographie, Dgat/Flash/Uac, Bénin, 94 P.

13. Mdaep, (2013) Accès À L'eau Portable Et À L'assainissement De Base. Rapport De Stratégies, Bénin, 112 P.

14. Odoulami, L. ; Boko, M. Et Togouet, Z. (2012). Assainissement Et Approvisionnement En Eau Potable Dans La Ville De Cotonou (Bénin, Afrique De L'ouest). In Acte Du $3{ }^{\text {ème }}$ Colloque Des Sciences, Cultures Et Technologies De L’uac-Bénin, Issn : 1840-5851, Pp. 567-578.

15. Oms, (1992). Evaluation Des Eaux De Surface Dans Les Communautés À Faibles Revenus. Ed. O. M. D. L. Santé, Genève, 97 P.

16. Schwartz, D. (1995). Méthodes Statistiques. Paris, 4 Ème Édition Flammarion, $318 \mathrm{P}$.

17. Solidarité Internationale, (2020). Etat Des Lieux D'une Ressource Vitale : Défis Et Solutions. Baromètre 2020 De L'eau, De L'hygiène Et De L’assainissement. 6e Édition, 55 P. 